for the proper performance of such important, delicate and responsible duties as those of true pharmacy. But instead of dispensing his own medicines the physician should insist upon a radical reform in the average drugstore, including the banishment of the soda fountain, cigar stand and all other excrescences. Many druggists are uneducated. But the remedy is a reasonable measure of compulsory education which the physicians and the public should insist upon. We have numerous schools of pharmacy, and many of them are excellent schools; but not one of the pharmacy laws of the forty States that have such laws requires a single day's attendance at any such school as a prerequisite to the license to practice pharmacy, because the public and the medical profession are so indifferent as not to demand it. Reputable and honest merchants, called druggists, wholesale and retail, may buy and sell drugs and chemicals about which they know little or nothing, frequently accepting and furnishing in good faith one thing for another. But the only protection against such serious blunders is the faithful application of skill and knowledge by the properly trained pharmacist. Druggists, wholesale and retail, may buy and sell drugs which are so old as to be unfit for use, and those who periodically and critically examine their supplies and throw away all medicines not in perfect condition can be only those whose training fits them to do this intelligently. The remedy must be the just recognition of the plain fact that the pharmacist is a scientific-tech. nical expert who performs special services for which he is entitled to professional fees, and not a mere merchant dependent upon commercial competition.

Without rational scientific pharmacy there can be no scientific medicine, for how can the physician learn anything definite about the action of any medicine which is not uniform? Medicinal preparations must not only be carefully and properly prepared from materials of verified identity and quality, and then standardized; but they must be protected against change, examined from time to time, and thrown away whenever they shall be found no longer perfect.

The new pharmacy should not be a "store." but an officine. It should be provided with all the necessury apparatus, reagents, and other facilities for scientifictechnical pharmaceutical work, which should include not only the preparation, examination, compounding and dispensing of medicines, but also urine analysis, and other microscopic and chemic examinations required by physicians, the preparation of bacteriologic culture media, etc. There should be no patent medicines and other nostrums in the officine of the new pharmacist, no tobacco, no soda fountain, no merchandise-nothing but legitimate medicines, surgical dressings and whatever else is requisite to enable him to parform his proper functions as the laboratory ex. pert and assistant to the physician and the common chemist of the community. This is no idle fancy picture of an unattainable ideal. It is the reasonable and proper demand of our present civilization. Such pharmacies can be maintained in every large city, and in numerous smaller cities. Not only that, but in places where it may be impracticable to maintain such an officine, divorced from the miscellaneous merchandizing of the average drugstore of today, there can still be a vast improvement made upon the prevailing system.

Will not the members of the American Medical Assocration declare in favor of compulsory pl ceutic education for the practice of pharmacy and for the divorcement of legitimate pharmacy from the commercial drug business; in the interest of the public welfare?

\section{PRESENT STATUS OF SERUM THERAPY.}

Presented to the Section on Materia Medica, Pharmacy and Therapeutics at the Forty-ninth Annual Meeting of the American Medical Association held at Denver, Colo., June 7-10, 1898.

\section{BY GEO. W. COX, M.D.}

$$
\text { CHICAGo. }
$$

The history of serum therapy is like the history of a day - a busy day -into which is crowded the memory of a thousand conflicting incidents, all so brief and fleeting in character as to be almost forgotten on the morrow. Many of us remember the "good old days" of thumb lancet and fever starvation, when serum therapy was not even a dream; most of us remember when the clouds first began to separate under the influence of scientific research, and all of us remember when the first sunbeam of actual discovery burst upon the world like a meteor's gleam. It must not be inferred from this that no hint of the underlying principles of serum therapy was ever scented until the present generation, for a suggestion of them was made nearly two hundred years ago, when the Turks are credited with practicing a crude form of inoculation against smallpox. The methods were so imperfect, however, and their success so limited, that the practice was allowed to die out, and many decades passed before Jenner revived the idea and applied it in the form of vaccination. Although another half century rolled by before the next great advance was made, it would be very unjust to the immediate followers of Jenner to say that they were content to confine the principles of vaccination to the prevention and treatment of a single disease. Many theories were advanced by them-the chief one being the once popular but now almost forgotten isopathic practice of the Germans-and much experimentation indulged in; but their gropings were wholly in the dark until a practical application of serum therapy was made possible by the first important discovery of Louis Pasteur.

This great scientist, while still young enough to be addressed as "mon cher enfant" by Biot, the discoverer of the phenomena of polarization, taking molecular asymmetry as a cue, had demonstrated the power of the tartrates under certain conditions to deflect polarized light. This led him to the immediate study and speedy discovery of the true cause of fermentation-the real beginning of Pasteur's many and varied achievements. Then followed in rapid succession the numerous and useful ideas now so familiar to every student of medicine, including the explanation of the cause and nature of infection, of contagion, and of the principles of prophylaxis and cure by vaccination. He also determined the evolution of disease germs, both in and out of the living organism; and finally rounded out his brief but fruitful scientific career by giving to the world the new treatment for hydrophobia. Thus by Pasteur was laid the foundation upon which the superstructure of serum therapy is now being erected. I mention this point in simple justice to the inventive genius of a great man.

All great, important discoveries, through their greatness, soon become so popular in the public mind that
their originators are quickly forgotten. We speak 
of the tubercle bacillus, the telephone and the diphtheria antitoxin as commonplace affairs that had been known from the most ancient times, and rarely stop to think of the men who spent years of earnest toil in their discovery and development. Richet has truly said that the first.course medical student of today is perfectly familiar with many phenomena which the wisest sages of fifty years ago could not understand. Lavoisier, Jenner, Galen, Celsus and Hippocrates all knew that fermentations existed and were quite familiar with their consequences; but they had no more conception of their causation than we of the present day have of the origin and proximate character of electric force. It was not until the year 1857 that the bacterial origin of fermentation was made known and means devised by which it could be produced, controlled or prevented at will. The world of commerce began to be enriched at once, for the wine disease, the beer disease and the silkworm disease each in turn received the attention of the young French physicist, with the result of placing them all under easy control. This would seem to be glory enough for one man, and it seems strange that anyone would bid for more; but the highest reward was yet to come in the application of the same theory in regard to the causation of disease in man. Pasteur was not a physician, and this makes it all the more remarkable that he should have been singled out by the fortunes of destiny to lead the hosts of medical bondsmen into the light of etiologic, pathologic and therapeutic sunshine. Thus, while the whole world acknowledges Pasteur as the "father of bacteriology," as it is known and practiced today, through this relationship he must forever be designated as the grandfather of serum therapy. The question naturally arises whether the present progeny is of sufficient merit to be considered a worthy offspring of so famous an ancestry.

There is a brief, uncertain period in the life of every youth, as well as in every young scientific problem, when the weathercock of prophecy seems to hesitate between the points of great usefulness and utter worthlessness. It is the purpose of this paper to show that serum therapy has passed this period of uncertainty and assumed a place among the staples of rational therapeutics. It will be noticed that under the general term of serum therapy I include medication by toxins, antitoxins, blood serum and vaccinsall of which are the outgrowth of the germ theory of disease and the direct result of applied bacteriology. My definition is, therefore, intended to be practical rather than scientific, and I trust the importance of this phase of the subject will be permitted to outweigh technical inaccuracy. In presenting the claims of this new system it is necessary to first point out its great value as a diagnostic agent. The incipient stage of some diseases, tuberculosis for example, is so mild and insiduous that its early detection is impossible by the usual methods; but by means of a bacterial product it may be disclosed, both in man and in the lower animals, even before its location in the organism can be definitely determined. The advantages of this are self-evident. In the human subject it permits appropriate treatment to be instituted before chronicity and complications render the case hopeless; while in the case of cattle it favors isolation or such other disposition of the stock as will insure the owner against pecuniary loss. If, therefore, serum therapy had nothing else to recommend it, its value in the early diagnosis of two such diseases as tuberculosis and typhoid fever would insure it a permanent place in the list of esteemed remedies. But its worth is not mensured by its diagnostic poweralone. Its well-known action as a prophylactic in some diseases is of the greatest moment, while its curative power in others can be accurately described by the one word "specific."

In the consideration of serum therapy in its practical application to the treatment of diseases, it seems both natural and fitting that the one to be mentioned first and with which all comparisons should be made, is that relentless and loathsome enemy of childhood:

Diphtheria.-To the vast majority of the thinking. physicians of today, the mere mention of diphtheria is suggestive of the remedy par excellence in every civilized land-antitoxin. To be sure, there is yet to be heard an occasional denial of the most absolutely demonstrated facts in the common affuirs of life. It is not necessary to burden you with a long list of figures and statistics relative to the grand, onward march of success in the antitoxin treatment of diphtheria. The evidence is so overwhelming in its favor, and the few failures and accidents so easily accounted for that but one conclusion is possible to the man whose senses are not wilfully closed to investigation and truth.

Tetanus.--Early in 1894, Carle and Rattone demonstrated that tetanus was an infectious disease, capable of being transmitted from man to the lower animals by inoculation; but it was reserved for Nicolaier to discover the bacillus of tetanus, a few months after the publication of Carle and Rattone's experiments. The bacillus itself, as well as the disease it creates, was extremely difficult to study, and while Brieger made the first important advance in the discovery of tetanin in 1896, it was three years later that Kitasaio first obtained the germ in pure cultures and gave to the world the first description of its biologic characters. Since that time scientists in all parts of the world have constantly vied with each other in the attempt to find a remedy that would destroy the. germ or its ptomains, and thus afford an efficient means of combating the distressing and fatal disease - tetanus. Naturally they turned to serum therapy for a realization of their hopes, and with what success their efforts have been crowned may be told in a few words. Blood serums are peculiar in their action, and but few of them are perfectly understood. Some of them, as the antidiphtheritic serum, exert their influence solely upon the ptomains, making them true antitoxins, while others, like the antistreptococcic serum, perform their work by destroying the germs themselves and thus putting a stop to the production of toxins. Others again, such as the antitetanic serum, do not seem to have much, if any, effect upon either germ or toxin, and yet from indisputable clinical evidence they possess an immense therapeutic value. Just in what way their good results are brought about has never been determined. Professor Roux of the Institut Pasteur has suggested that their action is of a protective character and is directed upon the tissue cells, fortifying them against tetanin, or the still more deadly toxalbumin described by Brieger and Fränkel. This explanation clearly accounts for the wonderful immunizing properties known to exist in the antitetanic serum, and is the strongest possible appeal for the use of this agent as a preventive. Not a single case of tetanus has ever developed after an adequate 
prophylactic dose of a reliable antitetanic serum was given. This fact, demonstrated in hundreds of experimental cases at the Institut Pasteur three years ago, has led to the adoption of a routine practice of immunization by surgeons in places where tetanus is known to be endemic. Quite recently a physician told me that he had just used a prophylactic dose of antitetanic serum after a rusty nail puncture, and the results were so satisfactory and his patient so free from apprehension during his illness that he should continue the practice, even if only for its moral effect.

The curative properties of antitetanic serum are negatively positive, lut not positively negative. It .cures-or more strictly speaking, it leads to recovery -by preventing any further invasion, but it does not cure the tetanus already existing. The only reason. able conclusion, therefore, is that the antitetanic serum should be used frequently at the first suspicion of an attack of tetanus, even before invasion if possible, or at least while the existing poison is not suffi. cient to cause death. While the serum treatment of tetanus is not all that could be wished for, nor all that we expect to see it in the future, as men and methods and means arise for its improvement, yet as we have it today it is by far the most successful treatment at our command. In Scotland, where statistics have been most carefully kept, the mortality has been reduced from the former frightful figure to about 35 per cent. In the United States, during the last two years, according to Dr. Frederic S. Dennis of New York, who has carefully investigated the subject, the mortality figure is even a shade lower still. Five cases of trismus nascentium were reported last year as successfully treated with the serum-cases which pre. vious to the days of antitoxin were almost uniformly fatal.

The experiments of Wasserman and Takaki, based upon Prof. Ebrlich's theory of the formation of antitoxins, should be remembered for the sake of future investigations in the general system of serum therapy. At present, however, they are interesting only from the laboratory standpoint and are absolutely without practical therapeutic value. More recently still, considerable local enthusiasm was excited in Rome, where Prof. Bacelli's treatment of tetanus with phenic acid was applied in thirty cases with but one death. This showing certainly entitles the method to further study; for while such a result in a single series of cases might be purely coincidental, the truly scientific mind should always stand ready to push investigation to its utmost limits when there appears the semblance of a cue. The very latest, and what appears to be the most reasonable, theory yet advanced concerning the action of the toxin and antitoxin of tetanus, is that of Roux and Borrel, in which they express the belief that the toxin attacks the nerve centers with such rapacity that the antitoxin, when administered hypodermically, does not reach the seat of destruction in time, or in sufficient quantity, to overcome the evil. They reasoned, therefore, that if the antitoxin could be applied directly to the cerebral substance, it might reach the vital point by rapid diffusion and be much more effective. In demonstration of this theory they tetanized seventy-nine guinea-pigs for their first experiments. Forty-five of these received intracerebral injections of small doses of antitoxin, and thirty-five of them recovered. Seventeen were treated with much larger doses by the hypodermic method, and of these only two survived. The remaining seventeen were not treated with antitoxin, and they all died. So far this method has only been applied in one case in the human subject. In this case the operation was entirely successful and the patient made a speedy recovery.

Tuberculosis.-Quite a number of toxins, antitoxins and blood serums have been introduced for the treatment of this disease, and with results that should be regarded as most gratifying, inasmuch as they have not all been absolute failures. The original tuberculin of Koch, and the improved product known as "T. R.", are admittedly most dismul failures as far as curative properties are concerned; but in spite of this they have wrought an excellent service in directing and stimulating investigation, as well as being of great value as diagnostic agents. Of the newer remedies of this class, as well as of the antitoxins and blood serums directed against the tubercle bacillus and its ptomains, the members of this Section will be fully informed in specially prepared papers relating to this subject. It remains, therefore, for me to discuss some of the complications of tuberculosis, and leave the consideration of the tubercle bacillus to the distinguished gentlemen who are making a life study of it and its terrible consequences. It has been so often repeated by various competent observers that simple, uncomplicated tuberculosis is easily curable, that it may seem strange to the casual reader that we hear of so few cures being made, The reason is that uncom. plicated cases are rarely met with in actual practice, the physician's services as a rule not being demanded until the disease has progressed far beyond the initial stage. Even then it is frequently found that the tubercular infection is the least consequential of the existing lesions. It is a well-known fact that unless tuberculosis is accompanied by some other pathologic condition, it usually remains localized for a long time, and the destruction of tissue from it alone is exceedingly slow. It is when other micro-organisms make their appearance that rapid tissue changes take place and the patient sinks quickly to the point of dissolution. To stop this process of rapid necrosis in the vital organs should be our chief aim, and in order to do so, it were better for us to forget for the time being that the tubercle bacillus has anything to do with the case, and give our entire attention to the organism that is doing the actual damage. In short, rid the case of its complicating influences first; convert it into a condition that is admittedly curable, and then direct our energies to the original disease. Can we always do this? Most assuredly we can not-but we can always try; and inasmuch as it is impossible for us to foretell the result of our efforts, it is clearly our duty to give our patients the benefit of the attempt. We will succeed in a certain number of cases; we will probably fail in many more, at least until effort and experience shall combine to suggest improvements in theory, methods and practice. In the light of past experience and present indications, however, it is reasonable to predict that if tuberculosis in its various forms and complications is ever to be placed in the category of curable maladies by means of med. ication, it will be accomplished by some method within the realm of serum therapy.

Snake bite.-The antitoxin used for the venom of poisonous reptiles is as surely specific in its action as any remedy known to medical science. The only drawback to its employment is the difficulty of procuring the serum at the required moment. The ab- 
sorption and action of the poison are so rapid that death is likely to result before the remedy can be obtained; but where it is possible to apply the serum within eighty or ninety minutes after the reception of the sting, no fears need be had as to the outcome of the case. The limitations of the application of the serum as a curative have led a number of investigators to seek for a reliable prophylactic or immunizing procedure, by means of vaccination or otherwise. Chief among such experimentors are Fraser and Phisalix, who have made exhaustive researches, and claimed decided success with such substances as bile, glycocholate of sodium, cholesterin and the tyrosin of certain vegetables. Calmette, however, whose name will always be associated with the antitoxin of snake venom, has shown conclusively that these substances only furnish a weak and transitory immunity by means of cell stimulation, the same as may be induced by a large number of others, and in no wise act as true antitoxins. So for the present, at least, the only known safeguard for persons living in regions infested by poisonous snakes is to make sure that a supply of the antivenomous serum is kept within easy reach.

Erysipelas,-The writer's experience in the treatment of this disease with blood serum, has been comparatively limited, but it has been so uniformly successful in a number of trying cases that he can not help thinking that if failure should be reported it must be due to the employment of faulty serum or some equally avoiclable cause. Marmorek's serum is the one indicated in all stages of the disease, and a number of cases reported by me at the annual meeting of the Illinois State Medical Society on the 17th of last month most conclusively show the immunizing power of the serum, as well as its specific action as a prophylactic and curative agent. The number of cases reported as successfully treated by the serum method already extends into the thousands, as every one interested in the collection of statistics can testify.

Yellow fever.- With the single exception of tuberculosis, there is probably no disease in which the bacteriologists of the Western hemisphere are more intensely interested, at the present time, than yellow fever. We are just at that critical period of anxious expectancy when any moment may witness the end of all dispute as to the question of causation, and the true germ of the disease be established beyond all cavil. And while we are thus waiting for the settlement of this one point, which we confidently hope will enable us to formulate a remedy that will place this dread disease in the column of preventable and curable maladies, it is fitting that we should allow our minds to dwell for a moment upon the names of those most intimately connected with this work. Among those in foreign lands, that of Sanarelli stands out with conspicuous luster, while in our own country we have a full half dozen of equally earnest and equally intelligent workers. Without detracting in the least from the fame of any of these, if asked to mention the man above all others, native or foreign, who is entitled to the palm for pioneer work in this field, for long, untiring and intelligent effort, as well as for modesty in presenting his just claims, we would unhesitatingly breathe the name of the present distinguished president of the AMERICAN MEDICal Association, George M. Sternberg. Placing the serum treatment of yellow fever upon the same rational and scientific basis as that of diphtheria and other infectious diseases, then, we can not expect the remedy to precede a knowledge of the etiologic germ. Whether this shall eventually prove to be the bacillus icteroides or the bacillus " $x$ " (Sternberg), if these are not identical, or some micro-organism yet to be discovered, remains to be seen. In the meantime the Sanarelli serum should be given a thorough trial, as pronounced success or pronounced failure would materially assist in settling the question of etiology.

Typhoid fever.-So far no serum has been prepared that can be implicitly relied upon as a cure for this disease, although several attempts have been made. The most promising one, at this time, is that of Prof. Chantemesse of the University of Paris, who claims to have had marked success in a number of recent trials--noting rapid defervescene and decided improvement in the general symptoms. $\mathrm{He}$ thinks, with a little more time for investigation and opportunity for the improvement in the preparation of the serum, it will take rank with those of well-established therapeutic value. In the matter of diagnosis, however, it is quite different, the Widal test being now regarded as a perfectly reliable and practical method for the busiest practitioner. It is possible to determine by means of this test, not only the very early invasion of typhoid fever, but also whether an individual has had the disease, even after the lapse of many years, in some cases. The test is of especial value on account of its accuracy and the simplicity of its technique.

In the brief space allowed for the discussion of this subject, it would be impossible to give the details of all that has been accomplished in the application of serum therapy to the various diseases and conditions in which it has been tried, however interesting it might be to do so. Up to this time, something more than a score of diseases have been subjected to treatment by this new method, and, as would naturally be expected, with varying success. In addition to those already mentioned, these include among others, pneumonia, influenza, rheumatism, cholera, oancer and sarcoma, pertussus, leprosy, gonorrhea, chancroid and syphilis, bubonic plague, anthrax, chorea and puerperal septicemia. A few of these deserve more than a passing notice, both on account of the prevalence and gravity of the diseases and the promising outlook for the early discovery of efficient remedies. Yersin and Haffkine have already succeeded in greatly mitigating the ravages of the bubonic plague, while numerous investigators have given us encouraging reports in regard to the probable successful management of leprosy in the near future. Sarcoma has undoubtedly yielded in a number of instances to the influence of the Coley mixture, while Asiatic cholera, the depopulating scourge of many nations and of all ages, seems destined to lose its terror through the beneficent agency of serum therapy.

Pneumonia.-Since the discovery of the bacillus of Friedlander in 1883, a host of bacteriologists, including Sternberg, Flügge, Talamon, Fränkel, Netter, Belfanti, Gameléia and many others, have studied this germ and the micrococcus pneumoniæ crouposæ with great diligence, in the hope of arriving at definite conclusions in regard to pneumonia and its proper treatment. It is generally found that mixed infection exists in this disease, and for this reason it will be necessary to pursue investigation and wait with such patience as we can command for the perfect success. 
that we hope to meet. Serums have already been used; and in some cases with gratifying success. Klemperer reported six cases with excellent results, and his experience has since been duplicated by Mosny, Bonne, Emmerich and Pansini. During an epidemic of pneumonia in the city of Naples, in the month of January of the present year, Pane treated nine cases of a very grave type with antipneumococcic serum. The effect of the remedy is reported as decidedly good in every case, and but one of the patients died.

Puerperal septicemia.-When caused by the streptococcus microbe, as it is in a very large proportion of cases in this disease, is no longer the frightfully fatal affection of bygone years, for it yields in a most gratifying manner to the antistreptococcic serum of Marmorek.

Streptococcal infection.-If this condition meant but a single disease, however severe, instead of a number of distinct maladies and a multiplicity of complications, its presence would not be regarded with more concern than that of tetanus or snake bite. But in all probability a score of such affections as these would fade into insignificance in comparison with streptococcal infection, if the full measure of its destructive virulence were known. We find it in all kinds and conditions of disease, sometimes as the sole pathogenic factor, but more often as an insidious and insinuating "co-respondent" adding dangers to those inherent in the original malady, or even supplying them where none existed before. Already the study of the streptococcus microbe has been productive of much valuable information. and a number of diseases can be traced directly to its presence; but inasmuch as its pathogenetic power is known to vary from absolute innocuousness to the most destructive virulence, there must yet remain much to be determined. The wide variation in its pathogenesis, its unapproachable extent of distribution, and the unusually attractive field it presents to the microscopist, all ecmbine to make its study both fascinating and instructive. Much confusion has been caused by the more or less arbitrary subdivision of the streptococcus into a large number of "kinds" or "species," bestowing such names upon each supposed new discovery as the fancy of the investigator would dictate. Koch, Marmorek, Widal and other high authorities, however, do not recognize any of the classifications thus far made, but regard all the so-called forms of the germ as the offspring of a single parent microbe, and attribute its varying pathogenic effects to such conditions as location, temperature, the kind and health of tissues involved, presence or absence of other micro-organisms, etc. Just why or how the same germ should cause convulsions in one animal and paralysis in another has never been explained, and possibly never will be, but as long as the same remedy, by equally mysterious methods, works a cure in both, we need not trouble ourselves about the pathogeny. Erysipelas, phlegmon and most cases of puerperal septicemia are caused by the streptococcus germ, and their certain and rapid cure by the proper use of Marmorek's serum is a fact so often demonstrated that it seems almost unnecessary to repeat the assertion.

However important it may be to know we have so efficient a remedy for these diseases, it is still of greater interest to know that the same remedy is equally successful in controlling certain complications that are more or less constant in many infectious dis- eases, including scarlet fever, diphtheria and tubercu. losis. Scarlet fever is probably always complicated by streptococcal infection; and in the experience of many close observers in Europe and America, when Marmorek's serum is administered early in the attack the disease is greatly modified, runs a mild course and the patient recovers without any disagreeable sequelæ. Streptococcal invasion is likewise the most constant complication attending cases of diphtheria; and no doubt many of the fatalities reported are due to this cause, and not to the toxemia produced by the Klebs-Loeffler bacillus, which may have been already overcome by the use of diphtheria antitoxin. Mixed infection should be suspected and looked for in every case of diphtheria, and when found a mixed treatment resorted to as early as possible. In this way the present extremely low mortality rate in this disease may be still further reduced, for without doubt nearly all of the fatal cases would show mixed infection if the proper tests were applied, and many more recoveries would occur if the proper (mixed) treatment was used.

It would be gratifying to me to hold a clinic at this time and present a number of tuberculous subjects, which have come under my observation during the last two years. They would all be cases of pulmonary tuberculosis with mixed infection, the predominating complication being of the streptococcal type. In at least two of these cases the complicating factor was so overwhelming in extent and severity that I am tempted to refer to them as cases of streptococcal infection complicated by tuberculosis. They had all the classic symptoms of well-advanced pulmonary consumption, and repeated examination of the sputum revealed the presence of the bacillus of tuberculosis, but always in small numbers. They had stubbornly resisted antituhercular treatment for many months; the direction of their progress was continually downward; faith had entirely deserted them, and hope was rapidly going. They were even denied the questionable consolation afforded by the promise of a lingering invalidism, for their physicians had named the limit beyond which they could not survive, and the date was almost reached. Microscopic examination of the sputum had heretofore been directed solely to the tubercular aspect-.. now it was made with reference to the complications. Streptococci in vast numbers were found. A complete change in the treatment was instituted, which was speedily followed by a most gratifying change in condition. All measures having reference to the tubercular infection were suspended and concentrated effort given to the streptococcal invasion. Marmorek's serum was given in 10 c.c. doses every alternate day until the streptococci all disappeared. By this time there was such an improvement in the general condition that hope was immediately revived and the patients put back upon the former treatment. One of them has remained constantly under my care, while the other, on account of distance from home, was returned to her former physician. In both cases the improvement continued; and now, after a lapse of nearly twenty months since the Marmorek's serum was stopped, my patient reports himself in better health than he has been for many years. Although there still remains a suggestion of tubercular toxemia, it is too slight to cause detention from business, and there has been no return of the streptococcal invasion.

Aside from the many flattering reports that come from all quarters of the globe in regard to the merits of serum therapy as a system, a careful study of its 
principles, and personal application of its methods, have convinced me that its vaunted worth has not been overstated. It is based upon reason, common sense, and truly scientific grounds, and its future development can not fail to shed untold blessings upon suffering humanity.

2945 Groveland Avenue.

\section{SOME SOURCES OF FAILURE IN TREATING LACHRYMAL OBSTRUCTIONS.}

Presented to the Section on Ophthalmology at the Forty-ninth Annual Meeting of the American Medical Association, held at Denver, Colo. June 7-10, 1898.

BY LEARTUS CUNNOR, A.M., M.D. DETROIT, MICH.

Accepted methods of treating lachrymal obstructions succeed in some cases but fail in others, for reasons not always apparent. Bearing on the sources of failure are the following suggestions, grouped under four heads: Constitutional disorder, eye strain, nasal disease and faulty technique.

First. It is evident that if lachrymal obstruction be caused by a chronic constitutional disease, the best planned and most skillfully executed operation will come to naught, for surgery never cured syphilis, gout nor rheumatism, and yet observation shows that this obvious truth is not infrequently overlooked. Spyhilitic infection may diplace the puncta, obstruct the canaliculi, induce a dacryocystitis, locate a mucous patch about the mouth of the duct, cause a periostitis, endostitis or subperiosteal gumma, adjacent to, or necrosis of the bony wall of, the duct; or hyperplastic processes of the bones themselves. These lesions may be found in any stage, from insignificant to gravest, singly or combined; but it is evident that operative treatment of obstruction due to any of them, would be useless unless accompanied by constitutional. More frequent though less amenable to general treatment are lesions of the tear passages, induced by gout or rheumatism or rheumatic gout and involv. ing the mucous, fibrous or bony tissues. Their management by purely operative procedures is quite as futile as surgical operations on deposits of these dyscrasia in other portions of the body, if disassociated from suitable diet, exercise, baths and general medi. cation. Tuberculous and scrofulous diseases do not always spare the lachrymal passages, and one who would do the best for every case of epiphora and record the fewest failures will not overlook the possibility of their presence under the most unexpected conditions. These are the most common chronic constitutional diseases, likely to produce lachrymal obstruction, though others may not safely be neglected. It has occurred to the writer to observe so many failures from exclusive operative measures which subsequently recovered under appropriate general treatment that he is compelled to emphasize the latter.

Second. It is generally accepted that eye strain causes or may cause conjunctivitis and blepharitis that blepharitis and conjunctivitis may induce inflam. mation of the lining membrane of the canaliculi and sac and lachrymal obstruction, so the conclusion is inevitable that eye strain may cause lachrymal obstruc. tion. In its earlier stages, epiphora from eye strain is readily cured by the treatment of the diseases of the conjunctive and lids, but like the latter, it returns with subsequent eye strain, while the correction of the eye strain is followed by permanent relief of both disabilities. Less happy is the exclusively operative treatment of lachrymal obstruction from eye strain, as the mutilation of the passages added to the eye strain renders the case even worse.

It is objected that eye strain is not always attended by epiphora. Quite correct; neither do all cases have blepharitis or conjunctivitis: nor would all of a thousand persons have pneumonia if suddenly plunged into an ice-cold stream, but some would. Unquestionably, there are conditions in certain individuals, which direct the disturbance of eye strain to the lachrymal passages, that are absent in others. Among these may be noted anatomic deformities of the bony walls of the duct. It is a clinical fact that lachrymal obstructions are more frequent in persons with a very broad or a very narrow-based nose. It is an anatomic fact that such faces have the bony wall of the duct flattened either anteriorly or laterally, so that the actual caliber of the passages is diminished and the more readily obstructed by morbid agents acting upon their lining membrane. It is not improbable that other congenital abnormalities determine the eye strain toward the lachrymal passages and cause obstruction. Further, it is not impossible that such deformities in some cases render failure inevitable from any or all treatment. We can lengthen a shortened leg by a mechanical contrivance, correct ocular defects in the shape of the eyeball, by lenses; but we may not hope to make round a bony lachrymal duct, greatly flattened in any direction.

Is it inquired how eye strain causes epiphora? Answer may be mado:

1. By direct extension of disease from the conjunctiva and edges of the lids, to the canaliculi and duct and sac. Retention of microbes in the lachrymal lake enables them to manufacture a larger volume of debris, as well as augment their own armies, conditions favorable for causing disease of the tear passages. 2. By disturbing the position of the puncta, through muscular action of the lids in efforts to overcome the refractive defect. 3. By interfering with the natural action of the lid muscles in propelling the tears through the canaliculi and sac to the duct. The clinical proof, that eye strain causes epiphora, and its relief stops it in certain cases, is abundant, but the scope of this paper does not permit of its presentation. If seen early in their course no other treatment than the relief of eye strain is needed; but later, when strictures have formed, or dacryocystitis, or other serious complications, surgical interference must be added to the removal of the eye strain. The practical point is, correction of eye strain will remove lachrymal obstruction in some cases and benefit all.

Third. Some failures to relieve epiphora are due to the overlooking of a morbid condition of the nares adjacent to and involving the opening of the duct into the nose. Clearly it is quite as essential to examine the terminal as the beginning of a continuous tube if we would remove all obstructions. The variety, frequency and gravity of nasal disease render it surprising that the duct is not occluded with greater frequency than is shown by clinical observation. We can not discuss the nature of the morbid condition of the nares or the means for their removal, but their importance is unlikely to be overestimated. Ophthalmic surgeons who do not treat nasal diseases need to be constantly on their guard to detect their presence, and refer the cases to one able to intelli- 